\title{
Norois
}

Environnement, aménagement, société

\section{Patrimoine fluvial, modernité et maritimité : un rapport en évolution}

Le cas Newcastle-upon-Tyne

River Heritage, Modernity and Maritimity: an evolving relationship. The case of

Newcastle-upon-Tyne (England)

\section{Cécile Renard-Delautre}

\section{OpenEdition}

Journals

Édition électronique

URL : https://journals.openedition.org/norois/5824

DOI : $10.4000 /$ norois.5824

ISBN : 978-2-7535-5086-5

ISSN : $1760-8546$

Éditeur

Presses universitaires de Rennes

Édition imprimée

Date de publication : 31 décembre 2015

Pagination : 103-114

ISBN : 978-2-7535-5084-1

ISSN : 0029-182X

Référence électronique

Cécile Renard-Delautre, «Patrimoine fluvial, modernité et maritimité : un rapport en évolution », Norois [En ligne], 237 | 2015, mis en ligne le 31 décembre 2017, consulté le 28 janvier 2022. URL : http:// journals.openedition.org/norois/5824; DOI : https://doi.org/10.4000/norois.5824 


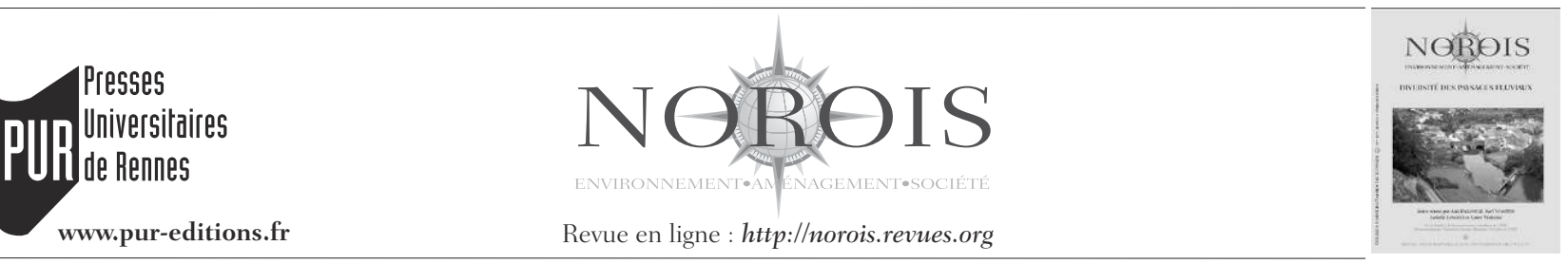

\title{
Note de recherche \\ Patrimoine fluvial, modernité et maritimité : un rapport en évolution Le cas de Newcastle-upon-Tyne (Angleterre)
}

\author{
River Heritage, Modernity and Maritimity: An Evolving Relationship \\ The Case of Newcastle-upon-Tyne (England)
}

\author{
Cécile Renard-Delautre
}

Architecte, Dr. Géographie, Chercheur associée EA EIREST. (Cecile.renard-delautre@univ-paris1.fr)

Résumé : Après la rudesse de la désindustrialisation, dans les années 2000, NewcastleGastehead a tenté de repositionner son projet de ville dans le registre culturel. Alors que l'on assistait au renouvellement d'une sensibilité au maritime, le réaménagement des quais centraux (projet Quayside) avait été pensé pour créer une vitrine métropolitaine du Tyneside.

Cependant, une quinzaine d'années après, ce tournant culturel n'a pas pris l'ampleur espérée et a notamment subi la crise de 2008. Les grands projets des années 2000 sont en partie rejetés par la population. Le patrimoine alors considéré comme une « ressource territoriale », est aujourd'hui au cœur de l'ancrage des habitants sur le territoire. Cet article revient sur l'évolution des dynamiques patrimoniales du Tyneside.

Mots clés : Tyneside - patrimoine fluvial - patrimoine industriel - tourisme - maritimité

Abstract: After the harshness of deindustrialisation, in the 2000's, NewcastleGastehead tried the repositioning of the city on a cultural concept. In a context of a new sensitiveness for the sea, the redevelopment of the central quays (Quayside project), was planned to become a metropolitan window of the Tyneside.

However, fifteen years later, this cultural turn is not as successful as expected and has notably suffered from the 2008 economic crisis. The iconic projects of the 2000's are partly rejected by the population. While heritage was considered as a "territorial resource", it is now central in the matter of "sense of place". This article deals with the evolution of "patrimonialisation" in the Tyneside.

Keywords: Tyneside - river heritage - industrial heritage - tourism- maritimity

\section{INTRODUCTION}

Comme son nom l'indique, Newcastle-« uponTyne » est une ville posée sur son fleuve, la Tyne River; en effet, sur l'autre rive, naît la ville de Gateshead. Le fleuve fédère la "Tyneside », cinquième agglomération la plus peuplée d'Angleterre.
L'histoire des interactions dynamiques entre Newcastle et la Tyne est complexe et marquée par de grandes étapes. Comme souvent, la ville est née de la présence de l'eau : à l'époque romaine s'établit Pons Aelius, une cité dotée d'un fort marquant l'extrémité est du Mur d'Hadrien, dont les ruines 
ont été inscrites au patrimoine mondial en 1987. Dès l'origine, les interactions entre la ville et son fleuve sont liées au commerce maritime car le port exporte du bois vers la Scandinavie et la Baltique. La vocation commerciale de la Tyne a perduré durant la période saxonne puis médiévale mais c'est avec l'exploitation massive des mines de charbon, au $\mathrm{XVII}^{\mathrm{e}}$ siècle, que la Tyne s'est révélée comme axe de transport majeur.

Situés au plus étroit de la gorge, à seulement quinze kilomètres de l'embouchure, les quais de chargement de Newcastle préservaient les navires des violences de la mer. Le fleuve était alors en permanence chargé de navires allant et venant de Newcastle vers le large et les chantiers navals de la Tyne atteignent leur apogée en tant qu' « ateliers du monde » (Moffat, 2006).

À l'instar de bien des villes industrielles de l'Amérique et de l'Europe du Nord, les années 1970 avec la désindustrialisation et la fermeture des mines ont progressivement conduit Newcastle à se détourner de son fleuve, l'urbanisme se focalisant sur des projets du centre-ville. Ce n'est que dans les années 2000 que la «waterfront attitude » (Gravari-Barbas, 1991; Bruttomesso, 1993; Smyth, 1994; Rodrigues-Malta, 2004) a gagné Newcastle. Le tournant culturel est alors engagé en suivant le modèle de Glasgow ${ }^{1}$ (Mooney, 2004). Trois constructions phares sont inaugurées sur les quais (le Millenium bridge, le Baltic, réhabilitation d'un silo à grain des années 1930 en musée d'art contemporain et le Sage, auditorium conçu par l'agence internationale d'architecture Foster \& Partners), propulsant le site sur la scène culturelle de la globalisation (Renard, 2012; 2013).

1. Dans les années 1990, Glasgow, à $200 \mathrm{~km}$ de Newcastle, fait figure de précurseur pour enrayer le "syndrome Germinal » (expression utilisée in "L'effet fierté », Libé-Villes, supplément de Libération du 11-12 décembre, 2004). Elle prend alors une valeur de modèle pour les villes du Nord de l'Angleterre, comme le résume ainsi Gerry Mooney : « elles parlent désormais de devenir les nouvelles Barcelone ou Bilbao, mais en réalité, les villes du Nord de l'Angleterre veulent être les nouvelles Glasgow » (Mooney, 2004). Glasgow avait pris de l'ampleur grâce aux chantiers navals sur les berges de la rivière Clyde et, à l'instar des villes post-industrielles, s'était dégradée, paupérisée pour devenir le décor sinistre du film Trainspotting, (Danny Boyle, 1996). Lasse de la crise qui se prolonge, des industries qui ferment toutes peu à peu et du chômage, la force de Glasgow a été de réagir et de refonder son " projet de ville » sur la culture (Masboungi, 2004). Cette refondation s'est concrétisée notamment par l'accueil de la manifestation Ville européenne de la culture en 1990. La candidature de Glasgow au titre de ville culturelle a surpris car elle rompait le cycle engagé par la manifestation qui n'élisait jusqu’à lors que des villes à l'héritage artistique incontesté (Athènes, Florence ou Amsterdam). La manifestation Glasgow, City of Culture 90 a été un véritable succès permettant à la ville de transformer durablement son image. «Doing a Glasgow » est d'ailleurs devenu une expression utilisée dans les traités de politique culturelle (Mooney, op. cit.).
Cet article a pour but de montrer l'évolution de la valorisation du patrimoine du Tyneside. Véritable «"ressource territoriale" associée aux développements du tournant des années 2000, ce patrimoine a bénéficié du renouveau d'une sensibilité au maritime » (Péron et Rieucau, 1996; Prelorenzo, 2011). Les interfaces ville-fleuve et ville-mer (Chaline [dir.], 1994) ont été repensées mais ces projets, et notamment celui des quais centraux de NewcastleGateshead (Qayside), ont eu du mal à être appropriés par les habitants. Comment ce patrimoine est-il alors mobilisé pour recréer du lien entre la population et le territoire? La valorisation patrimoniale institutionnelle (top down) suffit-elle à recréer ce lien? Comment une patrimonialisation bottom up émerge-t-elle?

Dans un premier temps, nous présenterons le cas de Newcastle et en particulier le tournant culturel des années 2000. Dans un deuxième temps, nous nous concentrerons sur la définition du patrimoine fluvial dont il est question à Newcastle. Enfin, nous analyserons les appropriations et dynamiques patrimoniales locales.

L'article s'appuie sur l'actualisation d'un travail d'analyse approfondi effectué par l'auteur en 2007 (Renard, 2007, non publié) ainsi que sur différents travaux de recherche (Bailey et Miles et al., 2004; Miles, 2005; Bailoni, 2008). La méthodologie repose sur une enquête de terrain qui comprend des entretiens qualitatifs avec les responsables du service du patrimoine de la ville de Newcastle, les représentants de English Heritage in the North East, les responsables de structures de transmission patrimoniales telles que le Discovery Museum, ainsi que les responsables et membres d'associations patrimoniales locales impliquées dans la préservation et transmission du patrimoine maritime (The Net).

\section{LE CAS DE NeWCASTle, ENTRE INTELLIGIBILITÉ LOCALE ET APPRO- PRIATION DE MODÈLES URBAINS}

\section{Attractivité, singularisation et modèles urbains}

Depuis le tournant des années 1980, les modes d'actions sur l'urbain ont été bouleversés par les mutations socioéconomiques (désindustrialisation), combinées avec la nouvelle étape de la mondialisa- 
tion. Dans ce contexte, on observe qu'une transversalité entre les politiques culturelles, patrimoniales et urbaines s'établit progressivement. Un brouillage s'opère entre les modes et les degrés d'actions sur les territoires : entre urbanisme, politique culturelle, marketing territorial et événementiel. À cela s'ajoute, une attention renouvelée pour le patrimoine, définie par François Hartog comme l'entrée dans un nouveau régime d'historicité dit «présentiste » (Hartog, 2003), qui transforme les actions de patrimonialisation tant «top down » que « bottom up ».

Si ce brouillage observé sert une logique d'attractivité, le renouveau des politiques patrimoniales s'explique également par l'appréhension du patrimoine comme une « rente de monopole » (Harvey, 2008 : p. 43-52). En effet, les stratégies de différenciation territoriale tentent de se fonder sur la valorisation des éléments uniques et non reproductibles.

Le contexte de la globalisation et de la libéralisation de l'économie a introduit une compétitivité entre les territoires. À différentes échelles, internationale ou régionale, cette concurrence interurbaine sur le plan économique, renforcée par un processus d'urbanisation toujours croissant, implique la nécessité pour les villes de se démarquer, afin d'attirer les investissements et, plus largement, de mobiliser des ressources financières (Veltz, 1996).

Si les villes cherchaient autrefois à se vendre en tant que lieux de production, elles se pensent désormais comme lieux de consommation (Harvey, 1989b). Ainsi, l'attractivité des territoires a évolué ces vingt dernières années, pour se tourner vers les flux touristiques afin, non plus de créer de la richesse, mais d'en capter un maximum (Davezies, 2004 ; Terrier, 2006). Afin d'attirer des capitaux par le biais des touristes (de loisirs ou d'affaires) ou par le biais d'une nouvelle population (de créatifs ou de gentrifieurs) - les villes rivalisent avec, comme armes, l'originalité de leur bâti et la plus-value de leurs équipements.

Toutefois, une observation des stratégies de singularisation urbaine fait apparaître le risque d'une uniformisation de modes d'intervention sur l'espace public en dépit de caractéristiques pourtant très variées. En effet, si les problématiques se ressemblent, une normalisation des réponses est à redouter. Cette idée de réponse "systématique» a été exprimée par Greg Richards (2011) à propos du tourisme culturel : l'auteur énonce l'application de « recettes », voire une « reproduction en série » des stratégies urbaines de différenciation : «In fact, many places follow similar strategies in order to achieve their uniqueness, which ends up making those places feel and look the same. This is a problem of "serial reproduction" is clearly evident in the development of many products related to cultural tourism, which includes elements such as: Iconic structures (e.g. Bilbao Guggenheim); Megaevents (e.g. European Capital of Culture); Thematization (e.g. Cultural routes); Heritage Mining (e.g. Historic quarters)» (Richards, 2009, p. 11) 2 .

Le tourisme culturel, envisagé comme une nouvelle manne économique qualitative, devient en effet l'objectif pour lequel se mettent en place ces stratégies urbaines de différenciation. Aux enjeux similaires, des réponses génériques apparaissent dans les esprits des acteurs urbains, galvanisés par les « success stories » telles que Bilbao ou Glasgow. Ainsi modélisée, la reconquête des fronts d'eau apparaît bel et bien comme un processus de production d'un lieu de «syncrétisme culturel » (Ruggeri, 2014).

Ainsi, la fabrication de ces ressources territoriales avec intentionnalité des acteurs (Gumuchian et Pecqueur, 2007 : p. 80-147) implique l'émergence de modèles plus ou moins appropriés localement. Cette intentionnalité est caractéristique d'un retournement de la relation entre l'économie urbaine et l'urbanisme, au tournant des années 2000. En effet, tandis que pendant des siècles, l'innovation et le développement urbains ont été le résultat de l'activité économique des villes, ils en deviennent désormais sa condition préalable (Gospodini, 2002 : p. 60). En ce sens, Newcastle upon Tyne est une parfaite illustration de cette tendance.

\section{Le tournant culturel de Newcastle-upon-Tyne (2000)}

Newcastle semble bien avoir appliqué stricto sensu les procédés énoncés. Si Chaline, considère en 1994, le waterfront de Newcastle comme une opéra-

\footnotetext{
2. «En fait, de nombreux lieux suivent les mêmes stratégies pour asseoir leur unicité, ce qui aboutit à leur ressemblance. C'est un problème de "reproduction sérielle" qui apparaît clairement dans le développement de nombreux produits liés au tourisme culturel, qui inclut des éléments tels que : les structures iconiques (i.e. le Guggenheim de Bilbao) : les mégaévénements (i.e. les capitales européennes de la culture); la thématisation (i.e. les routes culturelles); l'exploitation du patrimoine (i.e. les centres historique) », traduction personnelle.
} 
tion « achevée ou largement avancée » (Chaline, op. cit., p. 36), c'est sans compter avec le projet postérieur de Quayside, soit le réaménagement des quais pour en faire la nouvelle vitrine métropolitaine du Tyneside.

Ce vaste projet avait pour ambition de recentrer l'activité économique et l'animation de Newcastle et Gateshead aux abords de la Tyne. En 2005, Quayside représentait alors «la possibilité d'un futur optimiste dans une ère pessimiste » (Miles, 2005, p. 923) bien que des critiques se soient déjà élevées concernant le risque d'une " greffe » urbaine éloignée de l'identité et du sentiment d'appartenance locale (Bailey, Miles et al, 2004 ; Miles, op. cit.).

Trois étendards de ce projet urbain, trois icônes servent l'imagerie et la communication de ce projet urbain (figure 1 [planche I] et 2) : a) le Sage, un auditorium, véritable «starchitecture » (GravariBarbas et Renard-Delautre, 2015), réalisée par l'agence d'architecture Foster \& Partners (pour un coût d'environ 63 millions d'euros), b) le Millenium Bridge, passerelle piétonne blanche très iconique (100 millions d'euros) et enfin, c) le Baltic, réhabilitation d'un d'une ancienne minoterie en musée d'art contemporain (30 millions d'euros) ${ }^{3}$.

Pour accompagner ce nouvel essor, l'accueil d'événements de grande envergure a été envisagé (Chaudoir, 2007). En 2005, la Tyneside accueille la Tall Ships' Race ${ }^{4}$ qui donne l'occasion à Newcastle de faire la démonstration de sa capacité à organiser un événement de grande envergure, en s'appuyant sur ses équipements iconiques récemment inaugurés. NewcastleGateshead avait déjà accueilli la course en 1993 mais l'édition de 2005 vise cette fois à illustrer le net dépassement des formes traditionnelles de la maritimité (Péron et Rieucau [dir.], op. cit.) et à s'ancrer dans le festivisme contemporain. Le tournant culturel et le renouveau d'un rapport à la Tyne sont donnés à voir au monde par le biais de la médiatisation de l'évènement. L'interface entre NewcastleGateshead et la Tyne se revendique bel et bien comme nouveau territoire de la mondialisation

3. Chiffres issus de Miles, 2005.

4. La Tall Ship Race est une course annuelle de grands voiliers écoles (de 9 à plus de 100 mètres) organisée par Sail Training International dans les eaux européennes. L'un des objectifs de cette course est de rassembler les jeunes européens et d'encourager la formation à l'art de la navigation. À chaque édition, différentes villes-port sont choisies comme étapes. Cette sélection se fonde sur plusieurs critères liés à la capacité technique de l'accueil (équipements et infrastructures portuaires, profondeur d'eau, etc.) mais aussi sur la proposition d'un programme culturel dédié.

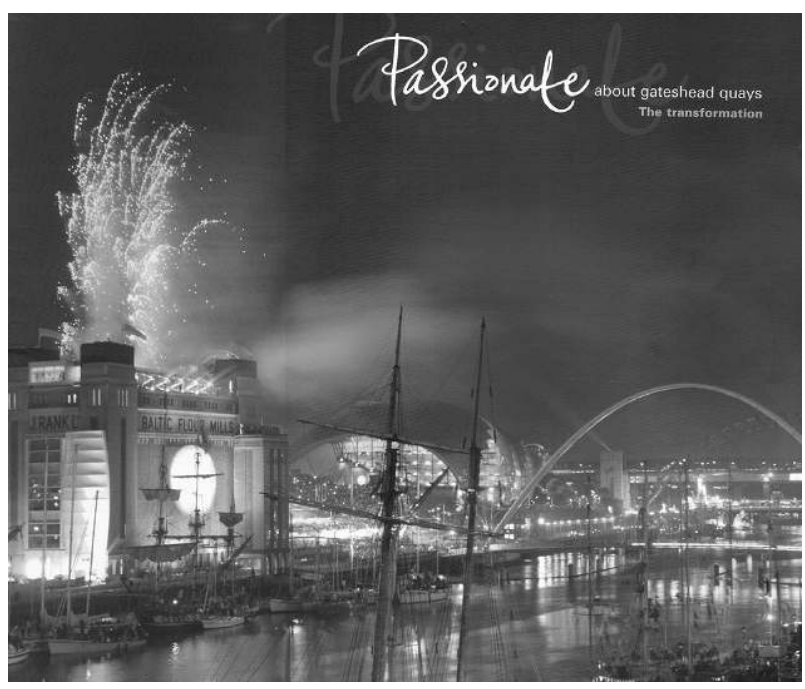

Figure 2 : L'imagerie de Quayside lors de l'accueil de la Tall Ships' Race (2005) (Source : Couverture de l'ouvrage de présentation du projet Quayside, Gateshead City Council, 2006)

(2005) The imaging of Quayside during the Tall Ships' Race

De gauche à droite : le musée d'art contemporain du Baltic, le Sage et le Millenium Bridge.

L'imagerie d'une «maritimité festive » créée par l'accueil de la Tall Ships' Race de 2005 sert la communication du projet de transformation des quais (projet Quayside). Sur cette photographie, « la fête » semble à son comble avec l'illumination des bâtiments et le feu d'artifice. L'impression d'une forêt de mâts sur la Tyne, d'ordinaire désertée par les navires, est accentuée par le premier plan.

(Baudoin, Collin, Prelorenzo, op. cit.), qu'il communique à grand renfort d'une imagerie qui tente de transformer les représentations locales.

Si la Tall Ships' Race est un succès ${ }^{5}$, se voulant le premier jalon d'une dynamique événementielle, l'impulsion est freinée par l'infructueuse candidature face à Liverpool pour le titre de Capitale européenne de la culture 2008.

En termes d'espace publics, le projet de Quayside s'est attaché ainsi à la réhabilitation des quais qui, dans les années 1990, n'étaient plus que des sites industriels vétustes où des grues à l'abandon menaçaient de s'écrouler. Les équipements étaient majoritairement restés en place mais l'activité avait déserté et les infrastructures se délabraient peu à peu.

Le projet de Quayside, les embellissements qu'il a engendré ainsi que la construction de la vitrine

5. Entre le 25 et le 28 juillet 2005, la Tall Ships' Race 2005 a attiré 1,5 millions de visiteurs à NewcastleGateshead, soit 500000 de plus qu'en 1993.

Le musée du Baltic (qui hébergeait le centre opérationnel de la course) a triplé le nombre de ses visiteurs (32000 entrées) durant cette même semaine (sources : «Tall Ships put Tyneside firmly in the spotlight », The Journal du 5 août 2005). 
métropolitaine composée par les trois icônes architectoniques (Millenium Bridge, Baltic et Sage) est le "flagship ${ }^{6}$ (Smith, 1994) d'un plan de régénération plus vaste partiellement suivi d'effets. Ce plan régional avait pour ambition de redynamiser l'économie ${ }^{7}$ mais aussi de mettre en valeur le paysage urbain de la gorge de la Tyne, dans l'optique d'une exploitation touristique, la prise de conscience de la valeur du fleuve et du front d'eau ayant, bien entendu, tenu une place décisive dans le projet.

Ce plan est à l'origine de la création d'une nouvelle entité artificielle à travers la création de la structure de promotion «NewcastleGateshead » en 2000. Une alliance improbable pour un programme de redéveloppement commun guidé par la culture. C'est d'ailleurs sous le nom de NewcastleGateshead que les deux villes ont concouru au titre de Capitale européenne de la culture 2008.

Le plan prévoyait des redéveloppements industriels et commerciaux au sud-ouest bénéficiant des espaces libérés par les anciennes industries. Il envisageait également l'expansion d'activités de loisirs, de petits commerces mais aussi de logements sur les quais centraux à l'endroit où le fleuve est le moins large et où les deux rives sont en tension. Tous ces aménagements devaient consacrer de nouveaux usages et marquer le retour de la population. La réhabilitation des quais a permis de redescendre vers le fleuve les fonctions administratives qui étaient jusqu'alors regroupées au nord, dans les hauteurs du centre-ville de Newcastle (figure 1).
6. Un flagship est un terme issu du vocabulaire naval qui a subit différents transferts : a) le champ du marketing s'est approprié ce terme dans un premier temps pour parler des produits-phares d'une marque; b) il a ensuite été associé à la création de magasins faisant office de publicité spatialisée pour de grandes marques (flagship store); c) enfin, le terme a migré dans le champ de l'urbanisme où il désigne un projet urbain ou un bâtiment (flagship urbain et flagship building) - vitrine du renouveau d'un territoire.

7. Entre 1978 et 1981, le chômage dans la région atteint $17 \%$ et concerne tous les secteurs économiques. Le redressement des deux villes entrâne leur mise en concurrence notamment dans des secteurs autrefois largement dominés par Newcastle. En 1986, l'ouverture du Metrocentre à Gateshead, qui est toujours aujourd'hui le plus grand centre commercial d'Europe, a contribué à réduire la suprématie du centre de Newcastle dans l'activité commerciale. D'une certaine manière, Gateshead a profité de la crise pour prendre une place plus importante dans l'économie régionale. À ces temps conflictuels entre villes voisines, a succédé, à partir de la fin des années 1980, le temps d'une réconciliation et la définition d'objectifs communs.

\section{« Nouvelle maritimité »: ENTRE USAGES, PATRIMOINE ET IMAGINAIRES}

Le patrimoine fluvial de Newcastle est issu de son passé industriel où la Tyne jouait son rôle de fleuveoutil, essentiel à l'activité économique.

Il existe un port sur la Tyne au niveau de Newcastle, depuis plus de deux mille ans mais c'est principalement avec l'exploitation massive des mines de charbon ${ }^{8}$, au XVII ${ }^{\mathrm{e}}$ siècle, que la Tyne s'est révélée un axe de transport indispensable. À seulement une quinzaine de kilomètres de l'embouchure, le fleuve était alors en permanence chargé de navires allant et venant de Newcastle vers le large. À partir des années 1850, la prospérité de la ville fondée sur le charbon, a permis d'étendre l'activité à de nouvelles industries (fer et acier) ainsi qu'à la production de fournitures liées aux expéditions maritimes (cordages, les ancres, les chaînes, les lampes), puis, au début du $\mathrm{xx}^{\mathrm{e}}$ siècle, aux productions de verre ainsi qu'à différents types d'industrie lourde et légère (moteurs pour les chemins de fer, armement, équipements miniers). Enfin, la production s'est ouverte aux produits chimiques. De tradition plus ancienne, les chantiers navals ont également connu une expansion à partir du XIX ${ }^{\mathrm{e}}$ siècle. La fin de la Seconde Guerre mondiale a marqué la fin de la suprématie de l'Angleterre dans la production de charbon ${ }^{9}$ mais aussi le déclin rapide des chantiers navals avec l'arrivée de nouvelles technologies et le développement du trafic aérien. L'effondrement progressif de ces industries a entraîné le chômage et la paupérisation de nombreux mineurs et ouvriers ${ }^{10}$.

À l'instar de nombreux autres territoires (Bird, $1963^{11}$, Chaline, op. cit.; Prelorenzo, op. cit.), les activités ont été déplacées vers l'embouchure, au début des années 1970, avec la création d'un nouveau port et d'un terminal en eau profonde. Ce déplacement a scellé la désertion des quais cen-

8. Le charbon a été extrait dès le XIII ${ }^{\mathrm{e}}$ siècle mais devient la base de l'économie de la ville à partir de la fin du XVII ${ }^{e}$ siècle.

9. Les mines du Northumberland et de Durham, ont continué à produire une grande quantité de charbon et à générer de l'emploi jusqu'à la fin des années 1960, date à laquelle l'industrie du charbon a connu une nouvelle crise avec les premières fermetures de mines en raison de l'épuisement des gisements mais aussi de l'émergence de nouvelles énergies (gaz, fuel et nucléaire).

10. Cf. le film Get Carter de Mike Hodges, 1971. Film à succès dont l'action se déroule à Newcastle. Le réalisateur a choisi de tourner le film dans cette ville pour accentuer la vision noire et dramatique de l'histoire.

11. Cf. le concept «d'anyport» (port quelconque) de James Bird, 1963 (représentation d'une évolution typique villes-ports britanniques). 
traux, jusqu’à leur réaménagement et l'avènement de nouveaux usages récréatifs.

Le réaménagement des quais encourage des usages tels que le jogging, le vélo, et grâce aux efforts de dépollution ${ }^{12}$, des pratiques de loisirs fluviaux comme la pêche ont pu se développer. En effet, « les sites au bord de l'eau favorisent l'émergence d'une nouvelle forme d'urbanité qui répond à la nouvelle sensibilité esthétique et écologique » (Prelorenzo, op. cit., §15). D’autres pratiques plus festives et nocturnes sont stimulées par la présence de bars et de discothèques. Un marché subsiste sous une nouvelle forme bien éloignée des anciens marchés de bouche (Milk market) et de fripes (Paddy market). Il n'attire plus la foule et il s'y vend " un peu de tout » (vêtements, artisanat importé, «souvenirs » divers, snacks). Depuis quelques années, ce «nouveau marché » bénéficie de "l'attraction» que constitue la mise en mouvement (bascule) du Millenium Bridge, les mercredis et les dimanches.

Si les quais connaissent ainsi un certain renouveau, on ne peut que constater, à l'instar de Claude Prélorenzo, que l'appropriation du fleuve (de l'eau) n’a, lui, pas su être réinventé : «On peut se demander s'il est possible de reconquérir des quais désertés et demeurés longtemps inaccessibles et de réinventer une nouvelle ville en relation avec l'eau, en laissant la rivière, le lac ou le bord de mer dans leur état actuel de stérilité qu'aggrave leur statut "d'espace libre" ou, plus vaguement encore, "d'espace naturel". Traiter un plan d'eau comme une place bleue, n'estce pas en dénaturer le sens et en ruiner la vitalité? » (Prelorenzo, op. cit., §15.) À Newcastle, le renouvellement de la maritimité semble s'être concentré sur l'aménagement du front d'eau, en misant sur la seule "force symbolique de l'eau dans l'image urbaine» (Garnier, 1995 : p. 274) et avoir ainsi délaissé l'usage de l'eau. Seule la Tall ships'Race, déjà mentionnée, a fait revivre temporairement le fleuve et a ravivé chez les plus anciens habitants le souvenir des bateaux

12. La pollution du fleuve a été une réalité dès l'expansion industrielle et en raison de l'augmentation des habitations le long des rives de la Tyne. Le système d'égouts victoriens n'était rien de plus qu'un collecteur qui se déversait directement dans la Tyne. Aucune amélioration n'a été entreprise avant la seconde moitié du $\mathrm{xx}^{\mathrm{e}}$ siècle. À cause du système particulier de flux et reflux du fleuve, les déchets déversés prenaient une dizaine de jours avant d'être évacués dans la mer. En 1966, un consortium des autorités locales a établi un Conseil chargé de régler la question de la pollution de la Tyne et de proposer un plan de gestion des égouts. Le schéma d'épuration de la Tyne, avec la construction de collecteur de part et d'autre du fleuve a été entrepris en 1973 pour n'être achevé qu’en 1993. Depuis les années 2000, la propreté de l'eau est néanmoins notable. qui autrefois faisaient partie du paysage quotidien de la ville.

Ces usages partiellement réinventés se fondent sur une nouvelle sensibilité au maritime qui se traduit tant par un regain d'intérêt des populations pour l'accès à la rive que par sa patrimonialisation. En effet, « espace identitaire, le fleuve constitue un élément de mémoire du lieu, un bien transmis, qu'il est question aujourd'hui à la fois de révéler, de rendre accessible et utilisable pour le plus grand nombre $^{13} »$ (Lechner, 2006, p. 5). C'est donc en termes à la fois de patrimoine (en tant qu'histoire et identité partagées) et d'imaginaires (en tant que construits) que s'est forgée progressivement cette sensibilité au maritime (Corbin, 1988).

Depuis une dizaine d'année, il semble que l'on assiste au changement de nature de ces imaginaires et du mobile de construction de la sensibilité au maritime. En effet, à l'univers fantasmagorique construit sur la base des vies des gens de mer et des industries qui en découlent, confronté à la rudesse du travail, s'est ajoutée une nouvelle forme de sensibilité fondée sur des valeurs esthétiques et des considérations environnementales. On assiste alors à la valorisation du fleuve en tant que patrimoine naturel et à l'émergence d'un imaginaire fluvial lié au cadre de vie. Le fleuve est ainsi présenté comme un « antidote urbain », ce qui se révèle dans les termes utilisés : «Anciennement nommée "friche", cette flore (et sa faune) est devenue "patrimoine naturel", précieux morceau de "sauvage" » (Romain, 2014, \$39).

Cette valorisation du rapport à la nature est à rapprocher de la tendance à l'imbrication des notions de patrimoine naturel et culturel (PA.TER.MONDI, 2014).

Si Newcastle n'est pas à proprement parlé au bord de la mer ${ }^{14}$ elle n'en subit pas moins la forte influence. Malgré une mer invisible depuis les quais centraux de la ville ${ }^{15}$, le caractère maritime du fleuve est prégnant.

13. Lechner, Gabriele, Le fleuve dans la ville, valorisation des berges en milieu urbain, Note de synthèse, Direction générale de l'urbanisme, de l'habitat et de la construction, Paris, Octobre 2006.

14. La Tyne se jette dans la mer du Nord à environ à 100 kilomètres de sa source, formée par la confluence de deux rivières, la North-Tyne et la South-Tyne. Depuis les quais centraux de la ville, l'embouchure se trouve à une quinzaine de kilomètres.

15. La direction de la mer est difficilement appréciable car le sens du courant, habituel indice, est modifié par les fortes marées. À Newcastle, l'amplitude des marées oscille entre deux mètres et quatre mètres cinquante. Le courant de marée est variable et sa vitesse peut atteindre un maximum de trois nœuds, soit $5,556 \mathrm{~km} /$ heure. 
Le système estuarien de la Tyne en fait un véritable corridor biologique, un patrimoine naturel que valorise la mise en place d'une signalétique le long des berges Nord et Sud de la Tyne. Elle indique notamment la grande variété d'habitats (depuis les forêts feuillues jusqu'aux prairies et aux marécages). Des plans d'action écologiques pour l'habitat de la gorge de la Tyne ont concerné la préservation de la végétation (broussailles, arbustes et haies), des forêts de feuillus, des paysages industriels ainsi que l'estuaire de la Tyne et les phénomènes de marnage. D’autres plans spécifiques visent la préservation de la faune constituée d'espèces telles que les loutres et la grive chanteuse musicienne. Ainsi, la mouette tridactyle (Kittiwake), qui contribue au caractère maritime de la Tyne, fait l'objet d'une attention particulière tant au niveau européen que britannique ${ }^{16}$. Le Tyne Bridge est d'ailleurs le plus important lieu de nidification artificiel du pays.

\section{Appropriations et DYNAMiQues PATRIMONIALES}

\section{Les leçons du tournant culturel en 2015 : ancrage local et polysémie patrimoniale}

Dans un article de 2005, Steven Miles (2005) faisait déjà le constat d'une interdépendance entre le succès de grands projets iconiques fondés sur la culture et l'importance de l'implication des habitants, regrettant que ce ne fut davantage le cas pour NewcastleGateshead. Dans cette lignée, Mark Bailoni remarque que Quayside n'est pas une entière réussite car le projet a mis de côté une partie de la population la moins aisée manquant son objectif de renouveau social et engendrant une gentrification (Bailoni, 2008, p. 11).

En 2015, le bilan reste mitigé et la situation stagne, voire s'aggrave, en raison des nouvelles difficultés économiques liées au contexte global de crise amorcée dès 2007-2008.

Au sein d'un Royaume-Uni en récession, la municipalité de Newcastle a lancé en mars 2013 différentes coupes budgétaires dont une réduction de la moitié des subventions culturelles, celles-ci passant

16. C'est une espèce protégée au niveau européen et britannique suivant la Convention de Berne de 1979, en annexe III et la EC Birds Directive de 1979 puis la Directive 2009/147/EC de 2009. Elle est également protégée par le Wildlife and Countryside Act de 1981. de 1,2 million à 600000 livres sterling ${ }^{17}$. De même, l'office de tourisme NewcastleGateshead Initiative, qui avait ouvert des bureaux dans le centre commerçant des hauteurs de Newcastle a été contraint de les fermer en avril 2015.

Les quais de Newcastle et Gateshead continuent de s'afficher comme une vitrine métropolitaine bien éloignée d'une reconquête populaire. Les fréquentations, les usages et pratiques des quais se sont renouvelés grâce aux aménagements et embellissements mais ne témoignent pas d'une appropriation exemplaire des lieux par les habitants. Le tourisme culturel, objectif phare du redressement de l'agglomération semble avoir désormais glissé vers la valorisation d'un tourisme de fête moins qualitatif. Les nombreux bars, restaurants et nightclubs attirent très largement une population régionale pour les weekends et les occasions spécifiques (Saint-Patrick, nouvel An, enterrements de vie de célibataires, etc.). La localisation des lieux de fêtes ne se situent pas spécifiquement aux abords du fleuve mais se répartissent dans le centre-ville de Newcastle ${ }^{18}$ (figure 1).

Dans le contexte d'un monde globalisé et hyper connecté, le patrimoine semble remplir une fonction d'ancrage local. S'il prend parfois l'aspect d'un repli identitaire, il représente aussi le besoin de replacer du sens et des valeurs au centre des modes de vies et correspond à une tendance générale de la société contemporaine (PA.TER. MONDI, op. cit.). Dans ce contexte "pro-patrimonialisation ", l'intérêt des populations locales pour le patrimoine ne fait que crôtre. En France comme au Royaume-Uni, les succès des Journées du patrimoine et Heritage Open Days (HODs) ${ }^{19}$ en sont un témoignage. Un rapport sur l'édition $2014^{20}$ indique que cette dernière a battu le record de l'année précédente au niveau national. À l'occasion des 20 ans de l'événement, différents prix et célébrations ont été remis dans le but de récompenser mais aussi de continuer à mobiliser les nombreux bénévoles. Le Tyne and Wear a ainsi été distingué pour la qualité de l'organisation, de la coor-

\footnotetext{
17. Source: Le Figaro du 8 mars 2013.

18. Observation participante de l'auteur.

19. À l'instar des journées du patrimoine, Heritage Open Days a lieu annuellement au Royaume-Uni chaque mois de septembre depuis 1994. Il s'agit d'une initiative du Conseil de l'Europe. Dans le Tyne and Wear, l'évènement de 2014 a été coordonné par les municipalités de Newcastle-upon-Tyne, de North Tyneside, South Tyneside et Sunderland.

20. Source : Heritage Open Days in Tyne and Wear Steering Group, Hertiage Open Days in Tyne and Wear 2014, Feedback report, novembre 2014.
} 
dination et de la promotion de l'événement. Depuis 2002, la région a ouvert un nombre croissant de sites (84 bâtiments et animations/18500 visites en 2002 et 172 bâtiments et animations/41 260 visites estimées en 2014) et se présente comme l'une des régions la plus dynamique du pays.

L'implication des bénévoles lors de HODs est significative d'une mobilisation notable de la population dans des initiatives de préservation et de mise en valeur du patrimoine.

Si ces initiatives isolées sont difficilement quantifiables, elles sont néanmoins au cœur de la préservation et la mise en valeur du patrimoine industriel et fluvial du Tyneside ${ }^{21}$. En effet, la municipalité de Newcastle considère que sa mission principale est la gestion et la préservation du patrimoine classé. Dans le contexte actuel de rigueur budgétaire, il lui est difficile d'outrepasser cette mission, en menant notamment des initiatives tournées vers le patrimoine industriel qui n'a pas forcément (encore) acquis cette reconnaissance patrimoniale institutionnelle ${ }^{22}$.

Dans les années 1990, dans une tendance générale de reconquête fluviale et de repositionnement du projet de ville, le service du patrimoine avait procédé à un inventaire de ce patrimoine à l'échelle du Tyne and Wear, donnant lieu à la publication d'un guide ${ }^{23}$. Dans cette mouvance quelques bâtiments avaient été classés par le système patrimonial national (English Heritage). Des réflexions avaient également pu être menées sur les stratégies de réaffectations, menant notamment à la décision de réhabilitation de l'ancienne minoterie en un musée d'art contemporain (Le Baltic).

Pour la municipalité, la question de la réaffectation des bâtiments est centrale car il est indispensable de réintroduire ce patrimoine bâti dans un nouveau modèle économique qui lui permette une autosuffisance. Suivant le type d'industrie concernée, le patrimoine est alors plus ou moins facile à réaffecter. L'exemple le plus extrême du Tyneside étant certainement les immenses structures de bois destinées au chargement de charbon

21. Source : entretien avec le directeur du service du patrimoine de la municipalité de Newcastle-upon-Tyne (Urban design and Conservation Team), mars 2015 .

22. Idem.

23. Ayris, Ian et Linsley, Stafford M. (dir.), 1994, A guide to the industrial archaeology of Tyne \& Wear, Publication de Tyne and Wear Specialist Conservation Team, Development Department, Newcastle upon Tyne City Council. appelées «Staiths». Le cas de celles de Dunston ${ }^{24}$, considérées comme les plus grandes d'Europe, et classées par English Heritage (Grade II Listed) est révélateur. Restaurées et accessibles au public dès 1990 pour le Gasthead Garden Festival, la question de leur gestion et de leur entretien s'est néanmoins montrée problématique. Touchées par des incendies, elles ont finalement été préservées par l'intervention d'une fondation de sauvegarde (Building Preservation Trust), qui a notamment impliqué de nombreux bénévoles pour aider à la récolte de fonds privés, comme c'est souvent le cas pour le patrimoine britannique.

Depuis une trentaine d'années, la notion de patrimoine a connu des mutations qui semblent n'avoir de cesse que de s'accélérer. Liées aux transformations sociétales d'une nouvelle étape de la mondialisation ainsi qu'aux économies territoriales qui encouragent la valorisation des ressources patrimoniales (Gumuchian et Pecqueur, op. cit. ; Lazzarotti, 2000), ces mutations induisent un engouement sans précédent pour le patrimoine de la part du public et par réciprocité, de la part des décideurs locaux. Dans cette dynamique, la gamme patrimoniale impliquant des objets allant « de la cathédrale à la petite cuillère » (Heinich, 2009) a été largement dépassée. Aujourd'hui, l'élargissement du champ patrimonial se poursuit dans plusieurs directions mais il implique surtout une polysémie qui le rend difficile à circonscrire.

Sous la bannière "patrimoine culturel ", s'intègrent, outre les biens classiquement listés et classés par le système national de la patrimonialisation (Monuments Historiques, English Heritage), une pluralité d'artefacts culturels de différentes natures, d'objets tangibles et intangibles mais aussi et surtout, de biens dont la valeur se fonde uniquement sur leur seule dimension testimoniale. Les usages anciens, les mémoires et les traces d'un passé commun, sont patrimonialités par le biais de collection d'objets d'un quotidien en train de disparaître, de la préservation de bâtiments désaffectés ou par le recueil de récits enregistrés. La valorisation de ces patrimoines mémoriels à ancrage territorial est un phénomène récurrent qui renforce l'idée du « tout patrimoine » (Neyret, 2004) et d'une omniprésence des référentiels patrimoniaux.

24. Les Dunston Staithes ont été construites en 1893; elles ont été partiellement démantelées en 1980. 
Ce type de fondations (Trust) est autant de structures autonomes, souvent créées à l'initiative de la population qui se sent concernée par la sauvegarde de « son patrimoine ${ }^{25} »$. Elle permet de parer aux incapacités des autorités nationales et locales à gérer des biens non économiquement soutenables. La municipalité semble encourager largement une appropriation de ce patrimoine par les habitants mais n'a pas la possibilité technique et de moyens pour en assurer la coordination. Les seuls Heritage Open Days, dont le programme est centralisé par l'agglomération, permettent une forme de recensement des initiatives portées localement.

\section{DES PATRIMONIALISATIONS TOP DOWN (LE DisCOVERY MuSEum) ET ВOTTOM UP (THE NET)}

Le Discovery Museum est un musée municipal des sciences et de l'industrie fondé en $1934^{26}$, dans une période contemporaine de l'ère industrielle de la région. Ainsi, le musée se positionne sur un registre qui n'est pas clairement patrimonial mais davantage sur l'idée d'une continuité entre l'histoire et le présent de Newcastle et de ses environs. À l'instar de la conception française d'un écomusée ${ }^{27}$, son objectif est de transmettre aux habitants et aux visiteurs l'identité et les fondements du développement économique et culturel local.

Pourtant, au fil de la constitution des collections, la distance entre les objets présentés et les habitants s'est creusée. Si, autrefois, ceux-ci connaissaient l'usage de certains outils, les visiteurs en sont aujourd'hui complètement éloignés; cette distanciation déplace les collections vers un registre patrimonial et testimonial. Ici encore, la participation de bénévoles est revendiquée et le recueil de témoignages sur la vie, les pratiques ou le maniement

25. Dans un contexte où la frontière entre patrimoine culturel et naturel a tendance à se brouiller (PATER.MON.DI, 2014), ces initiatives de préservation liées au bâti sont à mettre en parallèle avec des dynamiques de patrimonialisation de l'environnement du Tyneside et de son écosystème (ex. Tyne River Trust).

26. Le bâtiment qui accueille le musée a été construit pour the North East Cost Exhibitation (sorte d'exposition universelle britannique) de 1929.

27. En référence à la définition élaborée par Georges Henri Rivière pour la 9 e conférence internationale des musées de 1971. L'écomusée en France est une institution culturelle assurant sur un territoire donné les fonctions de recherche, de conservation, de présentation et de mise en valeur d'un ensemble de biens naturels et culturels représentatifs d'un milieu et des modes de vie qui s'y sont succédés. L'idée d'écomusée de Georges Henri Rivière fut expérimentée à partir de 1968 dans les parcs naturels régionaux français, puis en 1971 au Creusot. de certains outils est recherché (fig. 3a). Le musée cherche à faire vivre l'histoire locale, à rapporter des «petites histoires » qui forment la grande histoire.

Dépassant encore cette tendance, le musée a entrepris depuis quelques années la collecte d'objets contemporains. Si pour le moment, ceux-ci n’ont pas encore intégré les présentoirs du musée, ils sont néanmoins conservés dans cette optique ${ }^{28}$.

Le Discovery Museum possède une galerie spécifiquement dédiée à l'explicitation de l'essor de la région grâce au fleuve. "Story of the Tyne » rassemble des maquettes de bateaux construits dans les chantiers navals du Tyneside, des objets issus des productions de verre, les plans et maquettes des ponts qui enjambent la Tyne, des machines élaborées par les usines, etc. À l'entrée de la galerie cette phrase est inscrite sur le mur, pour rappeler (ou convaincre) qu' «aujourd'hui, la Tyne reste l'élément vital de la région ${ }^{29} »$.

Si la galerie expose l'essor industriel, le propos relate également les aspects sociaux en témoignant de la rudesse des métiers de mineurs, d'ouvriers sur les chantiers navals ou en présentant les petits métiers disparus.

En 2004, une fresque composée de portraits anciens et contemporains d'habitants a été exposée (figure 3b), l'objectif étant d'illustrer une forme de continuité de valeurs du territoire.

Le tournant culturel est également présenté, mettant notamment en valeur l'accueil du grand événement maritime qu'a été la Tall Ships' Race de 2005. Une annonce immobilière pour un appartement sur Qayside est exposée comme pour attester, montrer à la population, le renouveau économique du Tyneside.

Cette patrimonialisation institutionnelle (top down) tente de produire du lien entre les habitants et le territoire. La collection de témoignages et d'un « petit patrimoine » s'apparente aux actions menées par les populations elles-mêmes (bottom up) et illustre une tendance générale qui renouvèle la fabrique du patrimoine $^{30}$. Malgré ces efforts de la part des institutions pour faire émerger et mettre en valeur ce patrimoine

28. Entretien avec le conservateur de l'histoire maritime du Discovery Museum, mars 2015.

29. «Today, the Tyne remains the life blood of the region".

30. Voir, parmi bien d'autres exemples, le cas d'Hôtel du Nord à Marseille, détaillé dans cet article : Jolé, Michèle, 2012, "Hôtel du Nord. La construction d'un patrimoine commun dans les quartiers nord de Marseille », Métropolitiques, 4 janvier 2012. 

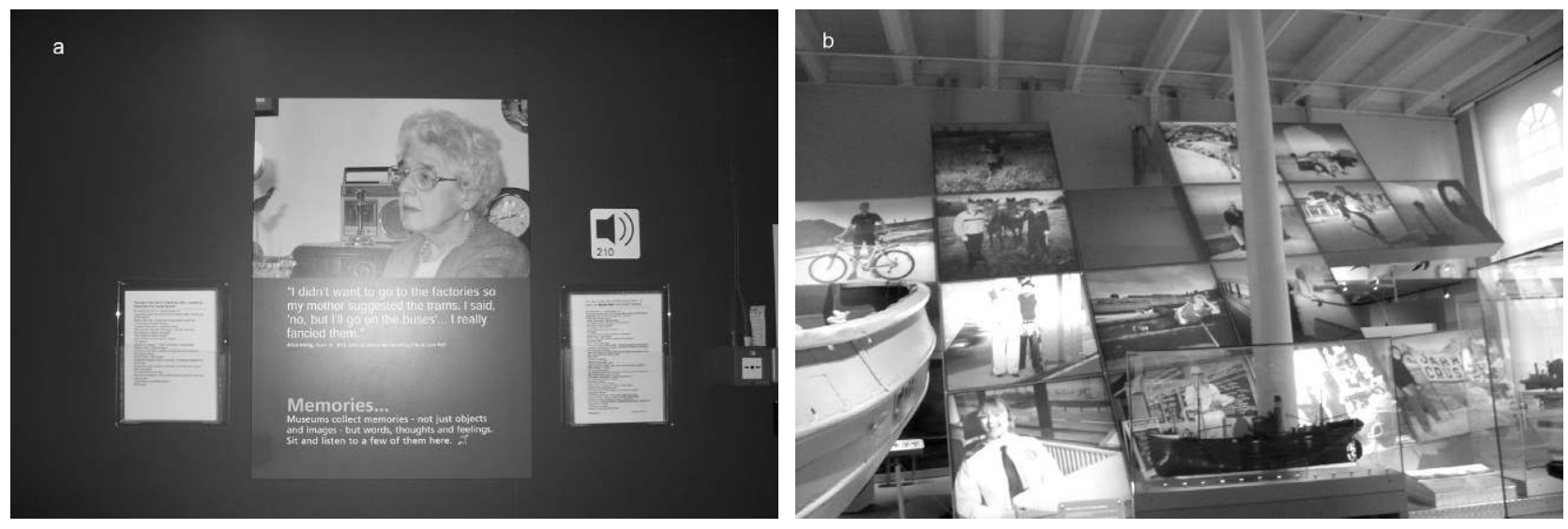

Figure 3 : L'idée d'une continuité identitaire au Discovery Museum The idea of an identity continuation at Discovery Museum

a) Le recueil de témoignages est recherché. Des panneaux incitant les habitants à livrer le témoignage de leur vie passée sont exposés. b) La fresque de portraits anciens et contemporains d'habitants de Newcastle évoquent les pratiques et les usages des quais, actuels et passés.

local, des habitants éprouvent la nécessité de « coproduire » eux-mêmes leur patrimoine.

Ainsi, le projet du NET est l'un des exemples d'une initiative bottom up à l'œuvre dans le Tyneside. Situé à North Shields, à l'embouchure, son objet principal est de préserver le patrimoine maritime local, notamment à travers l'héritage des pêcheurs locaux. L'objectif est de constituer une collection et de la mettre en visite dans les locaux réhabilités à cet effet dans le bâtiment du Old Low Ligt House (figure 4c). Grâce aux dons et à l'implication de nombreux bénévoles, le lieu a pu ouvrir sa collection aux visiteurs à partir d'avril 2015.

The Net emploie deux personnes permanentes dont la directrice (muséographe de formation); la structure est également gouvernée par un bureau exécutif. Un groupe de recherche (Old Low Light Research Group), composé de bénévoles (amateurs ou professionnels) est chargé de la collecte des objets et des témoignages en lien avec le patrimoine maritime ainsi que de leur inventorisation (figure $4 \mathrm{a}$ et b).

La démarche de constitution de la collection est celle d'une « coproduction» du patrimoine par les communautés locales et les gestionnaires du site ${ }^{31}$. En effet, l'objectif affiché est de rompre avec une traditionnelle dynamique top down des structures «patrimonialisantes» telles que les musées. Ici, si l'idée est de centraliser la mémoire du passé maritime, cela ne se conçoit pas sans une implication totale de la population. Cette démarche correspond

31. Entretien avec la directrice du Net. à l'idée d'un «sens du lieu » (sense of place), en lien avec une conception britannique de l'écomusée défendue notamment par Peter Davis, professeur émérite à l'université de Newcastle (Davis, 2011) ${ }^{32}$.

\section{Conclusion}

Le Tyneside doit son essor à son fleuve grâce auquel l'agglomération a connu une période faste et dynamique. La Tyne était alors un axe de transport majeur sur lequel les navires allaient et venaient. Les quais étaient soumis à la même effervescence et se présentaient comme le centre névralgique de la région. Après la rudesse de la désindustrialisation, les acteurs locaux ont tenté de redynamiser les abords de la Tyne et de replacer le fleuve au centre de l'activité urbaine. À travers le repositionnement du projet de ville dans un registre culturel et festif, NewcastleGateshead a tenté de réinvestir les usages et les imaginaires marins associés à la Tyne. Dans cette optique, le patrimoine industriel a été en partie mis en valeur, comme en témoigne l'iconique réhabilitation de l'ancienne minoterie musée d'art contemporain (Baltic). Cependant, comme l'avaient déjà remarqué différents auteurs dès 2004 (Bailey et Miles et al., 2004; Miles, 2005; Renard, 2007; Bailoni, 2008), il semble que ce projet n'ait pas suscité une appropriation de la part de toute la population du Tyneside. Au contraire, une partie d'entre elle ne s'est pas reconnue dans ces développements rapides, iconiques qui ont finalement soulevé

32. Entretien avec Peter Davis. 

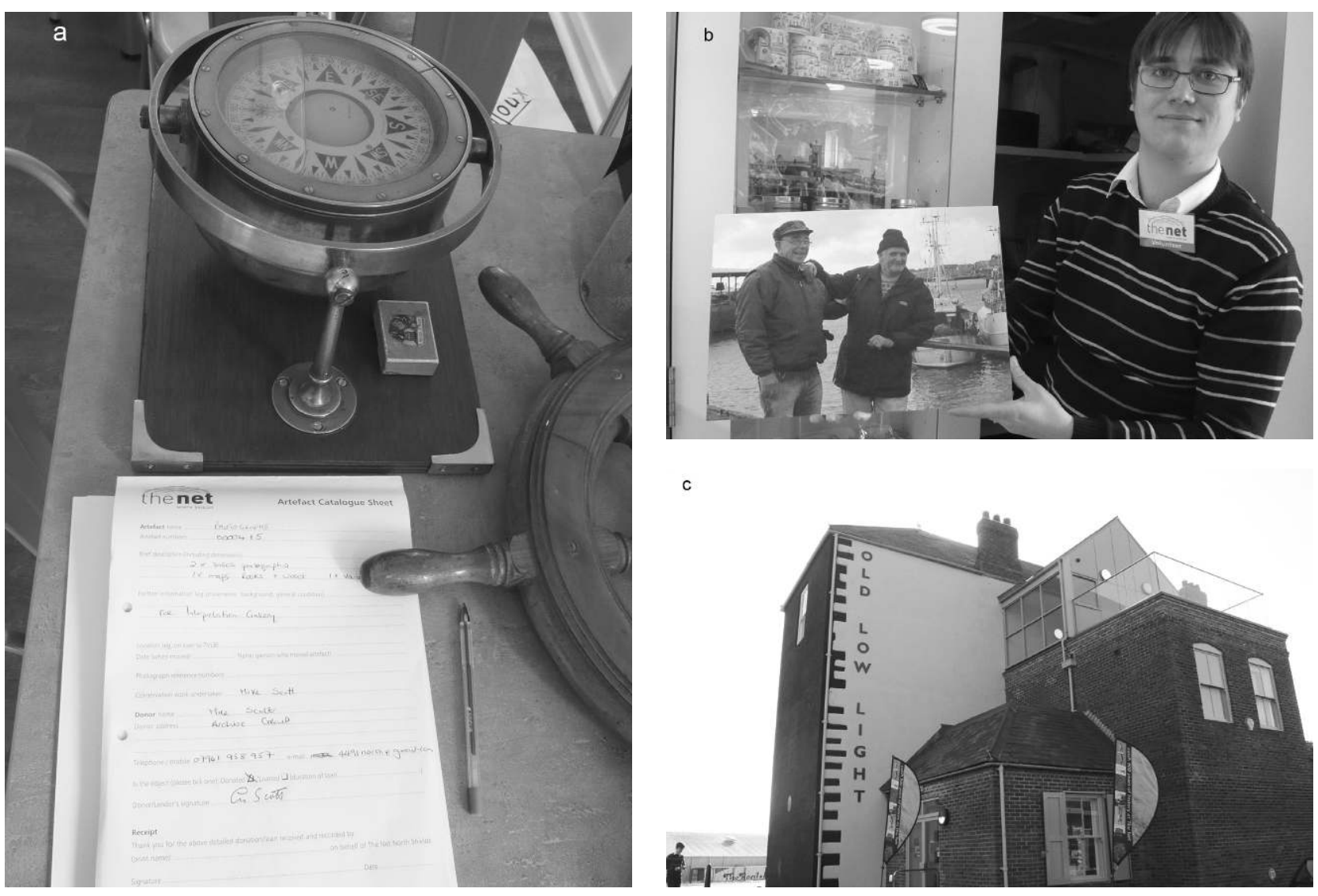

C

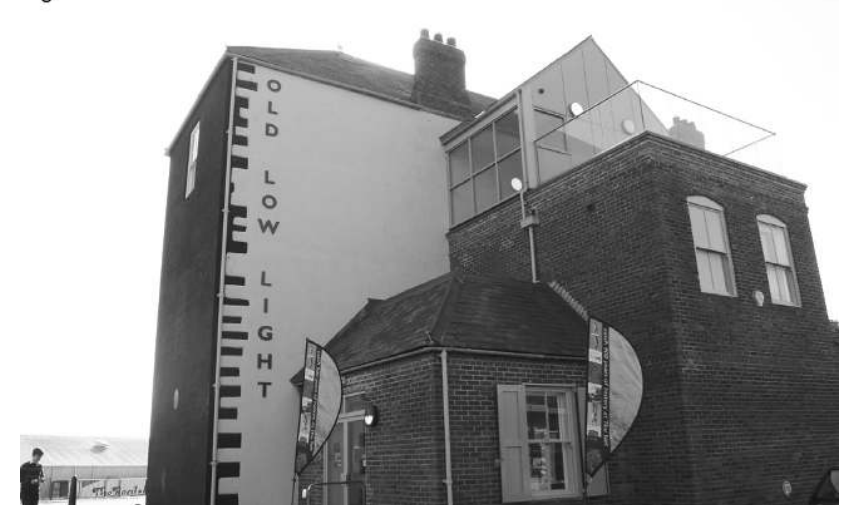

Figure 4 a, b et c : Le projet NET

The NET project

a et b) Le principe de constitution de la collection se fonde sur des dons. Chacun peut y apporter des objets qui lui semblent appartenir à cette mémoire martitime. Puis, le processus de classification et inventaire est fait par les bénévoles. Paul, un bénévole venu d'Allemagne, présent depuis le démarrage du projet, aide ainsi the Net à inventorier les objets. c) «Catch 900 years of History at the Net » annoncent les drapeaux à l'entrée du Old Low Ligt House.

une forme de rejet. Une quinzaine d'années après, ce tournant culturel des années 2000 n’a pas pris l'ampleur espérée. Subissant comme bien d'autres territoires la crise de 2008, les développements culturels et touristiques ont fait face à d'importantes coupes budgétaires qui n'ont pas permis de dynamique. Le tourisme culturel, qui avait notamment été annoncé comme un objectif, peine à trouver son public tandis que l'office de tourisme « Newcastle Gateshead » a été contraint de fermer ses portes en en avril 2015. Le secteur touristique se trouve alors modifié et se satisfait d'une fréquentation régionale, moins qualitative, centrée sur la fête (dont les célébrations liées au football).

La tendance générale montre une réappropriation des problématiques patrimoniales par les individus. Dans de nombreux contextes, les citoyens se sentent concernés par l'idée de recueillir, protéger et trans- mettre un patrimoine qui constitue une part importante de leur identité. Cette tendance se rencontre dans le Tyneside où elle s'apparente à une réappropriation d'un patrimoine fluvial qui semblait leur avoir échappé sous l'effet des politiques de marketing territorial. Les initiatives sont diverses. Elles impliquent de nombreux bénévoles pour qui il paraît essentiel de s'investir localement dans la transmission d'un passé révolu, mais aussi de pratiques sur lesquelles des «témoins » peuvent encore apporter un éclairage. Comme si le regret de ne pas avoir davantage pris garde à ce patrimoine auparavant se faisait sentir, on assiste à aujourd'hui à l'émergence de démarches qui ont pour objectif de «patrimonialiser le contemporain » : sauvegarder le présent pour mieux le transmettre demain. 


\section{Bibliographie}

Ayris I., Linsley Stafford M. (dir.), 1994. A guide to the industrial archaeology of Tyne E Wear, Publication de Tyne and Wear Specialist Conservation Team, Development Department, Newcastle upon Tyne City Council, 84 p.

Bailey C., Miles S., Stark P., 2004. Culture-led regeneration and the revitalisation of identities in Newcatle, Gateshead and the North of England, International Journal of Culture Policy, $\mathrm{n}^{\circ} 1$, vol. 10, p. 47-65. DOI:10.1080/1028663042000212328.

BAILONi M., 2008. Quelle place pour le patrimoine dans le renouveau d'une région postindustrielle? Le cas du NordEstanglais, Revue Géographique de l'Est, $\mathrm{n}^{\circ}$ 12, vol. 48 [https://rge.revues.org/682]

BIRD J., 1963. The Major Seaports of the United Kingdom, Londres, Hutchison, 486 p.

Bruttomesso R. (eds.), 1993. Waterfronts. A new frontier for cities on water, Venise, International center cities on water, $351 \mathrm{p}$.

Chadoin O., Godier P., Tapie G., 2000. Du politique à l'oeuvre: Bilbao, Bordeaux, Bercy, San Sebastián. Systèmes et acteurs des grands projets urbains et architecturaux, La Tourd'Aigues, Éditions de L'Aube, 240 p.

Chaline C. (dir.), 1994. Ces ports qui créèrent des villes, Paris, L'Harmattan, coll. « Maritimes », 300 p.

Chaudoir P., 2007. La ville événementielle : temps de l'éphémère et espace festif, Géocarrefour, $\mathrm{n}^{\circ}$ 3, vol. 82, p. 2-77.

Corbin A., 1988. Le territoire du vide : l'Occident et le désir du rivage, 1750-1840, Paris, Flammarion, $412 \mathrm{p}$.

Davezies L., 2004. Temps de la production et temps de la consommation : les nouveaux aménageurs des territoires?, Futuribles, $n^{\circ} 295$, p. 43-56.

Davis P., 2011. Ecomuseums: A Sense of Place, Londres, Bloomsbury Academic, 320 p.

Garnier C., 1995. L'eau et la valorisation du milieu urbain, in Plan Urbain, L'eau dans la ville : Bilan général d'un programme de recherche expérimentation, 1983-1994, Paris, Presses de l'École Nationale des Ponts et Chaussées, p. 257-288.

Gospodini A. 2002. European cities in competition and the new « Uses » of urban design, Journal of urban design, $\mathrm{n}^{\circ} 7$ (1), p. 59-73.

Gravari-Barbas M. Renard-Delautre C. (dir.), 2015 , Starchitecture(s), Figures d'architectes et espace urbain, Paris, L'Harmattan, 272 p.

Gravari-Barbas M., 1991. La mer retrouvée : Baltimore et autres reconquetes de fronts d'eau urbains, Thèse de doctorat de Géographie, dirigée par Jean Bastié, Université Paris 4, 3 volumes, $893 \mathrm{p}$.

Gumuchian H., Pecqeur B. (dir.), 2007. La ressource territoriale, Paris, Economica, coll. «Anthropos », $252 \mathrm{p}$.

Harvey D., 1989. From managerialism to entrepreneurialism: the transformation in urban governance in late capitalism, Geografiska annaler, no 71(b), p. 3-17.

Harvey D., 2008. Géographie de la domination, Paris, Les Prairies Ordinaires, $118 \mathrm{p}$.

Heinich N., 2009. La fabrique du patrimoine : de la cathédrale à la petite cuillère, Paris, Éd. de la Maison des sciences de l'Homme, coll. «Ethnologie de la France », 286 p.
Hartog F., 2003. Régimes d'historicité. Présentisme et expériences $d u$ temps, Paris, Seuil, $272 \mathrm{p}$.

Histon V., 2006. Unlocking the Quayside: Newcastle Gateshead's Historic Waterfront Explored, Tyne Bridge Publishing.

LAND USE CONSUltants, 2003. Urban landscape stydy in the Tyne Gorge, étude réalisée pour English Heritage, Londres, 216 p.

Lazzarotti O., 2000. Patrimoine et tourisme : un couple de la mondialisation, Mappemonde, $\mathrm{n}^{\circ}$ 57, p. 12-16.

Lechner G., 2006. Le flewve dans la ville. La valorisation des berges en milieu urbain, étude réalisée pour la Direction générale de l'urbanisme, de l'habitat et de la construction, Paris, $120 \mathrm{p}$.

Miles S., 2005. "Our Tyne": Iconic Regeneration and the Revitalisation of Identity in NewcatleGateshead, Urban Studies, $n^{0}$ 5/6, vol. 42, p. 913-926.

Moffat A., Rosie G. 2006. Tyneside: a history of Newcastle Gatehead from earliest times, Mainstream Publishing, $448 \mathrm{p}$.

Mooney G., Cultural policy as urban transformation? Critical reflections on Glasgow, European City of Culture 1990, Local Economy, 19: 4, p. 327-340.

Neyret R., 2004. Du monument isolé au «tout patrimoine », Géocarrefour, vol. 79, n 3, p. 231-237.

PA.TER.MONDI (Consortium pluridisciplinaire), 2014. Rapport final de l'Atelier de réflexion prospective "nouveaux défis pour le patrimoine culturel », ANR, mars 2014.

Péron F., Rieucau J. (dir.), 1996. La maritimité aujourd'hui, Paris, L'Harmattan, Géographie et Cultures, 336 p.

Prelorenzo C., 2011. La ville portuaire, un nouveau regard, Rives méditerranéennes, $\mathrm{n}^{\circ} 39$, p. 13-22.

Rodrigez-Malta R., 2004. Une vitrine métropolitaine sur les quais. Villes portuaires du Sud de l'Europe, Les Annales de la recherche urbaine, 97, p. 93-101.

Renard C., 2007. Escale fluviale, escale urbaine. Newcastle Gateshead, une marititmité retrowvée, diplôme de fin d'études d'architecture, ENS d'architecture Paris-Malaquais.

Renard C., 2012. Architecture, métropolisation, globalisation. Le processus de globalisation à travers le paysage architectural. Lectures croisées de Barcelone, Berlin et Rome, doctorat de géographie, Université Paris 1 Panthéon-Sorbonne, 532 p.

Renard C., 2013. «L'architecture globale », une lecture dynamique des territoires dans la globalisation, Bulletin de l'association de Géographes Français, vol., 90, n² 2, p. 127-141.

Romain F., 2010. Le fleuve, porteur d'images urbaines : formes et enjeux, Géocarrefour, vol. 85, n³ 3, p. 253-260.

Romain F., 2014. L'imaginaire fluvial contemporain : un antidote urbain?, Projets de paysage [http://www.projetsdepaysage. frll_imaginaire_fluvial_contemporain_un_antidote_urbain_].

Ruggeri C., 2014. "Repenser la ville portuaire », Urbanités, $\mathrm{n}^{\circ}$ 4, [http://www.revue-urbanites.fr/4-edito/].

Sмутн Н., 1994. Marketing the city-the role of flagship developments in urban regeneration, Londres, Ed. E\&F Spon, 18 p.

Veltz P., 1996. Mondialisation, villes et territoires : l'économie d'archipel, Paris, PUF, 264 p. 